\title{
Polynomial cycles in certain local domains
}

\author{
by
}

\author{
T. PEZDA (Wrocław)
}

1. Let $R$ be a domain and $f \in R[X]$ a polynomial. A $k$-tuple $x_{0}, x_{1}, \ldots$ $\ldots, x_{k-1}$ of distinct elements of $R$ is called a cycle of $f$ if

$$
f\left(x_{i}\right)=x_{i+1} \quad \text { for } i=0,1, \ldots, k-2 \quad \text { and } \quad f\left(x_{k-1}\right)=x_{0} .
$$

The number $k$ is called the length of the cycle. A tuple is a cycle in $R$ if it is a cycle for some $f \in R[X]$.

It has been shown in [1] that if $R$ is the ring of all algebraic integers in a finite extension $K$ of the rationals, then the possible lengths of cycles of $R$-polynomials are bounded by the number $7^{7 \cdot 2^{N}}$, depending only on the degree $N$ of $K$. In this note we consider the case when $R$ is a discrete valuation domain of zero characteristic with finite residue field.

We shall obtain an upper bound for the possible lengths of cycles in $R$ and in the particular case $R=\mathbb{Z}_{p}$ (the ring of $p$-adic integers) we describe all possible cycle lengths. As a corollary we get an upper bound for cycle lengths in the ring of integers in an algebraic number field, which improves the bound given in [1].

The author is grateful to the referee for his suggestions, which essentially simplified the proof in Subsection 6 and improved the bound for $C(p)$ in Theorem 1 in the case $p=2,3$.

2. Let $R$ be a discrete valuation domain of zero characteristic with finite residue field having cardinality $N(P)=p^{f}$. Fix a generator $\pi$ of the prime ideal $P$ of $R$ and denote by $v$ the norm (multiplicative valuation) of $R$, normalized so that $v(\pi)=1 / p$. Moreover, put $v(p)=p^{-\operatorname{ord} p}$. A cycle $x_{0}, x_{1}, \ldots, x_{k-1}$ will be called a $(*)$-cycle if $v\left(x_{i}-x_{j}\right)<1$ for $i \neq j$.

We shall prove the following results:

Supported by the KBN grant 210379101. 
THEOREM 1. (i) The length of a (*)-cycle in $R$ does not exceed $(N(P)-1) p^{C(p)}$, where

$$
C(p)=1+\frac{\log (\operatorname{ord} p)}{\log 2} .
$$

(ii) The length of a cycle in $R$ does not exceed $N(P)(N(P)-1) p^{C(p)}$, where $C(p)$ is given in (i).

In case of $R=\mathbb{Z}_{p}$ we can be more precise:

Theorem 2. (i) $A(*)$-cycle of length $n$ exists in $\mathbb{Z}_{p}$ if and only if $n$ is a divisor of $p-1$ except for $p=2,3$ in which case $n$ can be any integer not exceeding $p$.

(ii) If $p>3$ then a cycle of length $n$ exists in $\mathbb{Z}_{p}$ if and only if $n=a b$, where $a$ is a divisor of $p-1$ and $b \leq p$. The set of possible cycle lengths in $\mathbb{Z}_{2}$ is $\{1,2,4\}$, and in $\mathbb{Z}_{3}$ it is $\{1,2,3,4,6,9\}$.

COROLlaRY 1. Let $R$ be the ring of all integers in an algebraic number field of degree $N$ over the rationals. The cycle lengths in $R$ are bounded by $\left(2^{N}-1\right) 2^{N+1}$.

Corollary 2. If $k$ is the length of a cycle in $R$ then

$$
k \leq \min \left(N\left(P_{1}\right)\left(N\left(P_{1}\right)-1\right) N\left(P_{2}\right)\left(N\left(P_{2}\right)-1\right)\right),
$$

the minimum being taken over all pairs $P_{1}, P_{2}$ of prime ideals with

$$
\operatorname{char}\left(R / P_{1}\right) \neq \operatorname{char}\left(R / P_{2}\right) .
$$

For cyclotomic fields $K$ the bound given in Corollary 1 can be essentially improved:

Corollary 3. Let $K_{M}$ be the $M$-th cyclotomic field and $R$ its ring of integers. The cycle lengths in $R$ do not exceed $c_{4}(\varepsilon) M^{2 L+\varepsilon}$ for every $\varepsilon>0$, where $L$ denotes the Linnik constant.

Note that $N=\left[K_{M}: Q\right]=\varphi(M) \gg M / \log \log M$, and thus the cycle lengths in this case do not exceed $c_{5}(\varepsilon) N^{2 L+\varepsilon}$ for every $\varepsilon>0$, which is a much better bound than that resulting from Corollary 1.

3. We list first certain simple properties of cycles in arbitrary domains. We use the following convention: if $x_{0}, x_{1}, \ldots, x_{k-1}$ is a cycle, then for $n \equiv$ $r(\bmod k), 0 \leq r<k \leq n$ we put $x_{n}=x_{r}$. For $a, b \in R$ we write $a \sim b$ if $a$, $b$ are associated, i.e. differ by an invertible factor.

Lemma 1. Let $R$ be a domain and let $x_{0}, \ldots, x_{k-1}$ be a cycle in $R$ for the polynomial $F(X)=a_{n} X^{n}+\ldots+a_{1} X+a_{0}$. Then $k-1$,

(i) this cycle is a cycle for some polynomial $G$ of degree not exceeding 
(ii) if $a, b \in R, a$ is a unit in $R$ and $y_{i}=a x_{i}+b(i=0, \ldots, k-1)$, then $y_{0}, \ldots, y_{k-1}$ is a cycle for some polynomial over $R$,

(iii) if $k=r s$ then $x_{0}, x_{1 \cdot r}, \ldots, x_{(s-1) r}$ is a cycle for some polynomial,

(iv) for $0<r<k$ one has $\left(x_{i+r}-x_{i}\right) \sim\left(x_{j+r}-x_{j}\right)$,

(v) if $(i-j, k)=1$ then $\left(x_{i}-x_{j}\right) \sim\left(x_{1}-x_{0}\right)$,

(vi) if $x_{i}=a y_{i}, a, y_{i} \in R$, then $y_{0}, \ldots, y_{k-1}$ is a cycle for some polynomial.

Pr o of. (i) Take for $G$ the remainder of the division of $F$ by $\left(X-x_{0}\right) \ldots$ $\ldots\left(X-x_{k-1}\right)$.

(ii) The polynomial $G(X)=a F\left((X-b) a^{-1}\right)+b \in R[X]$ will do.

(iii) The sequence $x_{0}, x_{r}, \ldots, x_{(s-1) r}$ is a cycle for the $r$ th iteration of $F$.

(iv) Notice that

$\frac{F(X)-F(Y)}{X-Y}=a_{n}\left(X^{n-1}+\ldots+Y^{n-1}\right)+\ldots+a_{2}(X+Y)+a_{1} \in R[X, Y]$ and thus $x_{r}-x_{0}\left|x_{r+1}-x_{1}\right| \ldots\left|x_{k+r-1}-x_{k-1}\right| x_{r}-x_{0}$.

(v) In view of (iv) it suffices to deal with the case $j=0$. If $t>0$ is defined by $t \cdot i \equiv 1(\bmod k)$ then $x_{i}-x_{0}\left|x_{2 i}-x_{i}\right| \ldots \mid x_{t i}-x_{(t-1) i}$, hence $x_{i}-x_{0} \mid\left(x_{i}-x_{0}\right)+\left(x_{2 i}-x_{i}\right)+\ldots+\left(x_{t i}-x_{(t-1) i}\right)=x_{t i}-x_{0}=x_{1}-x_{0}$, but of course $x_{1}-x_{0} \mid\left(x_{1}-x_{0}\right)+\ldots+\left(x_{i}-x_{i-1}\right)=x_{i}-x_{0}$.

(vi) The $y_{i}$ 's form a cycle for $G(X)=a^{-1} F(a X) \in R[X]$.

\section{PROOF OF THEOREM 1}

4. From now on we assume that $R$ satisfies the conditions stated at the beginning of Subsection 2 .

Lemma 2. The length of any cycle in $R$ is a product of primes not exceeding $N(P)$.

P r o of. In view of Lemma 1(iii) it suffices to show that if $q$ is a prime exceeding $N(P)$ then there cannot be a cycle of length $q$ in $R$. Let $x_{0}, \ldots, x_{q-1}$ be such a cycle. In view of Lemma $1(\mathrm{v})$ one has $v\left(x_{i}-x_{j}\right)=v\left(x_{1}-x_{0}\right)=p^{-r}$ for $x_{i} \neq x_{j}$. Thus we can write $x_{i}=x_{0}+\pi^{r} w_{i}(1 \leq i<q)$ where $w_{i} \notin P$ and $w_{i}-w_{j} \notin P$ for $1 \leq i<j<q$, a contradiction.

LEMma 3. If $k$ is a cycle length in $R$ then $k=a b$, where $a$ is the length of some $(*)$-cycle in $R$ and $b \leq N(P)$.

Pr o of. Let $x_{0}, \ldots, x_{k-1}$ be a cycle. Assume first that for some $i>0$ we have $v\left(x_{i}-x_{0}\right)<1$, and denote by $b$ the smallest integer with this property. Then $b \mid k$. In fact, if $k=q b+r, 0<r<b$, then by Lemma 1(iv)

$v\left(x_{b-r}-x_{0}\right)=v\left(x_{(q+1) b}-x_{0}\right) \leq \max \left\{v\left(x_{(q+1) b}-x_{q b}\right), \ldots, v\left(x_{b}-x_{0}\right)\right\}<1$, contradicting the choice of $b$. 
It is obvious that either there exists a pair $1 \leq r<s<b$ with $x_{r}-x_{0} \equiv$ $x_{s}-x_{0}(\bmod P)$, and then $v\left(x_{s-r}-x_{0}\right)=v\left(x_{s}-x_{r}\right)<1$, which is impossible, or all differences $x_{r}-x_{0}(r=1, \ldots, b-1)$ are distinct $(\bmod P)$ and since they cannot lie in $P$ we get $b \leq N(P)$, as asserted. The numbers $x_{0}, x_{b}, \ldots, x_{(a-1) b}$ form a $(*)$-cycle.

5. Now we shall consider the lengths of $(*)$-cycles.

Lemma 4. Let $y_{0}, y_{1}, \ldots, y_{q-1}$ be a $(*)$-cycle of $F(X)=a_{n} X^{n}+\ldots+$ $a_{1} X+a_{0}, q$ prime, $y_{0}=0$. Then either $q \mid N(P)-1$, or $q=p$ and $a_{1}$ $\equiv 1(\bmod P)$.

Pr o of. Clearly

$$
\begin{aligned}
\frac{y_{k+2}-y_{k+1}}{y_{k+1}-y_{k}} & =\frac{F\left(y_{k+1}\right)-F\left(y_{k}\right)}{y_{k+1}-y_{k}} \\
& =a_{n}\left(y_{k+1}^{n-1}+\ldots+y_{k}^{n-1}\right)+\ldots+a_{2}\left(y_{k+1}+y_{k}\right)+a_{1} \\
& \equiv a_{1}(\bmod P)
\end{aligned}
$$

and thus

$$
1=\prod_{k=1}^{q} \frac{y_{k+2}-y_{k+1}}{y_{k+1}-y_{k}} \equiv a_{1}^{q}(\bmod P) .
$$

This implies

$$
a_{1}^{(q, N(P)-1)} \equiv 1(\bmod P)
$$

and hence $q \mid N(P)-1$ or $a_{1} \equiv 1(\bmod P)$.

Consider $a_{1} \equiv 1(\bmod P)$ and write $v\left(y_{1}-y_{0}\right)=p^{-d}$. Then

$$
\frac{y_{2}-y_{1}}{y_{1}-y_{0}} \equiv F^{\prime}(0) \equiv 1(\bmod P) \text {, }
$$

whence $y_{2}-y_{1} \equiv y_{1}-y_{0}\left(\bmod P^{d+1}\right)$, and similarly we get $y_{k+2}-y_{k+1} \equiv$ $y_{k+1}-y_{k} \equiv \ldots \equiv y_{1}-y_{0}\left(\bmod P^{d+1}\right)$. But then

$$
0=\sum_{k=1}^{q}\left(y_{k+1}-y_{k}\right) \equiv q\left(y_{1}-y_{0}\right)\left(\bmod P^{d+1}\right)
$$

and $q=p$ follows.

Lemma 5. Let $F \in R[X], g=F^{\prime}(0)$ and $a_{k}=F^{k}(0)$ with $v\left(a_{1}\right)=p^{-d}$, $d>0$. Then

$$
a_{k} \equiv\left(1+g+\ldots+g^{k-1}\right) a_{1}\left(\bmod P^{2 d}\right) .
$$

P r o of. Easy recurrence.

Lemma 6. If $m$ is the length of a $(*)$-cycle in $R$ and $p \nmid m$, then $m \mid N(P)-1$. 
Proof. Let $y_{0}, \ldots, y_{m-1}$ be such a cycle realized by $F$. In view of Lemma 1(ii), (vi) we can assume without loss of generality that $y_{0}=0$ and $y_{1}=\pi$. If we put $g=\left(y_{2}-y_{1}\right) /\left(y_{1}-y_{0}\right)$, then

$$
\frac{y_{k+1}-y_{k}}{y_{k}-y_{k-1}} \equiv g(\bmod P)
$$

and by Lemma 5 ,

$$
y_{k} \equiv\left(1+g+\ldots+g^{k-1}\right) \pi\left(\bmod P^{2}\right) .
$$

Suppose that for some $0<r<m$ we have

$$
y_{r} \in P^{2}
$$

and let $M$ be the smallest such $r$. Then $g^{M} \equiv 1(\bmod P)$ and $M \mid m$ since $y_{m}=0 \in P^{2}$. Let $v\left(y_{M}\right)=p^{-d}(d \geq 2)$ and write

$$
\underbrace{F \circ \ldots \circ F}_{M \text { times }}(X)=F^{M}(X)=b_{t} X^{t}+\ldots+b_{1} X+b_{0} .
$$

Since

$$
b_{1} \equiv F^{\prime}(0)^{M} \equiv g^{M} \equiv 1(\bmod P)
$$

we get

$$
y_{(k+2) M}-y_{(k+1) M} \equiv y_{(k+1) M}-y_{k M}\left(\bmod P^{d+1}\right)
$$

and

$$
0=\sum_{k=1}^{m / M}\left(y_{(k+1) M}-y_{k M}\right) \equiv \frac{m}{M}\left(y_{M}-y_{0}\right)\left(\bmod P^{d+1}\right)
$$

gives a contradiction.

Thus (2) does not hold and $y_{1}, \ldots, y_{m-1} \notin P^{2}$. If $m \nmid N(P)-1$, then $g^{m} \equiv 1(\bmod P), g^{N(P)-1} \equiv 1(\bmod P), g^{(m, N(P)-1)} \equiv 1(\bmod P)$ and using $(1)$ and remembering that $g \not \equiv 1(\bmod P)$ we get $y_{(m, N(P)-1)} \in P^{2}$, which contradicts the last statement.

6. By Lemmas 3 and 6 it remains to consider $(*)$-cycles of lengths $p^{\alpha}$.

Proposition. If there is a $(*)$-cycle of length $p^{\alpha}$, then $\alpha \leq C(p)$, where $C(p)$ is defined in Theorem 1.

Pr o o f. Let $x_{0}, x_{1}, \ldots, x_{p^{\alpha}-1}$ be a $(*)$-cycle. By Lemma 1 we can assume that $x_{0}=0$ and $v\left(x_{1}\right)=p^{-1}$. For $0 \leq k \leq \alpha-1$, put $v\left(x_{p^{k}}\right)=p^{-d_{k}}$ (so in particular $\left.d_{0}=1\right)$, and $\lambda_{k}=\left(F^{p^{k}}\right)^{\prime}(0)$. So for $k \leq \alpha-1$ one has

$$
1=\prod_{l=1}^{p^{\alpha-k}} \frac{x_{(l+1) p^{k}}-x_{l \cdot p^{k}}}{x_{l \cdot p^{k}}-x_{(l-1) p^{k}}} \equiv\left(\lambda_{k}\right)^{p^{\alpha-k}}(\bmod P) \quad \text { and } \quad \lambda_{k} \equiv 1(\bmod P) .
$$

Write $\lambda_{k}=1+u_{k} \pi^{w_{k}}$, where $u_{k} \notin P, w_{k} \geq 1$, putting $w_{k}=\infty$ in case $\lambda_{k}=1$. 
Lemma 5 gives

$$
x_{p \cdot p^{k}} \equiv\left(1+\lambda_{k}+\ldots+\lambda_{k}^{p-1}\right) x_{p^{k}}\left(\bmod P^{2 d_{k}}\right) .
$$

If $\lambda_{k}=1$ then for $d_{k+1}<2 d_{k}$ one has $d_{k+1}=d_{k}+$ ord $p$, and if $\lambda_{k} \neq 1$ then

$$
x_{p \cdot p^{k}} \equiv \frac{\left(1+u_{k} \pi^{w_{k}}\right)^{p}-1}{u_{k} \pi^{w_{k}}} x_{p^{k}}\left(\bmod P^{2 d_{k}}\right),
$$

leading to

$$
\begin{aligned}
x_{p \cdot p^{k}} \equiv\left(p+\left(\begin{array}{l}
p \\
2
\end{array}\right) u_{k} \pi^{w_{k}}+\ldots\right. \\
\left.\quad \ldots+\left(\begin{array}{c}
p \\
p-1
\end{array}\right)\left(u_{k} \pi^{w_{k}}\right)^{p-2}+\left(u_{k} \pi^{w_{k}}\right)^{p-1}\right) x_{p^{k}}\left(\bmod P^{2 d_{k}}\right) .
\end{aligned}
$$

Hence if $d_{k+1}<2 d_{k}$ then $d_{k+1} \geq \min \left(d_{k}+\operatorname{ord} p, d_{k}+(p-1) w_{k}\right)$ and we arrive at

$$
d_{k+1} \geq \min \left(2 d_{k}, d_{k}+\operatorname{ord} p, d_{k}+(p-1) w_{k}\right) .
$$

By putting $k=\alpha-1$ we get

$$
\begin{aligned}
p+\left(\begin{array}{l}
p \\
2
\end{array}\right)\left(u_{\alpha-1} \pi^{w_{\alpha-1}}\right)+\ldots+\left(\begin{array}{c}
p \\
p-1
\end{array}\right)\left(u_{\alpha-1} \pi^{w_{\alpha-1}}\right)^{p-2} \\
+\left(u_{\alpha-1} \pi^{w_{\alpha-1}}\right)^{p-1} \in P^{d_{\alpha-1}} .
\end{aligned}
$$

If $w_{\alpha-1}(p-1) \neq \operatorname{ord} p$ then

$$
d_{\alpha-1} \leq \operatorname{ord} p
$$

Otherwise

$$
w_{\alpha-1}(p-1)=\operatorname{ord} p .
$$

For $k \leq \alpha-2$ one has

$$
\lambda_{k+1}=\left(F^{p^{k+1}}\right)^{\prime}(0)=\prod_{j=0}^{p-1}\left(F^{p^{k}}\right)^{\prime}\left(x_{j \cdot p^{k}}\right) \equiv \lambda_{k}^{p}\left(\bmod P^{d_{k}}\right),
$$

and thus we obtain

$$
w_{k+1} \geq \min \left(d_{k}, w_{k}+\text { ord } p, p w_{k}\right) .
$$

In the case $p=2$ we need stronger inequalities. Since

$$
\lambda_{k+1} \equiv \lambda_{k}\left(\lambda_{k}+\left(F^{p^{k}}\right)^{\prime \prime}(0) x_{p^{k}}\right)\left(\bmod P^{2 d_{k}}\right),
$$

and $2 \mid\left(F^{p^{k}}\right)^{\prime \prime}(0)$ the inequality

$$
w_{k+1} \geq \min \left(2 d_{k}, w_{k}+\operatorname{ord} 2,2 w_{k}, d_{k}+\operatorname{ord} 2\right)
$$

results.

Lemma 7. For $k=0,1, \ldots, \alpha-1$ one has $\min \left(d_{k}, w_{k}\right) \leq$ ord $p$. 
Proof. If the assertion failed for some $k$, then (3) and (6) would imply

$$
w_{\alpha-1}, d_{\alpha-1}>\text { ord } p
$$

contradicting (4) and (5).

Lemma 8. For every prime $p$ and for $k=0,1, \ldots, \alpha-1$ one has

(i) $d_{k} \geq 2^{k}$ in case $d_{k} \leq$ ord $p$,

(ii) $w_{k} \geq 2^{k-1}$ if $p$ is odd,

(iii) $w_{k} \geq 2^{k}$ if $p=2$.

Proof. First consider the case of $p \neq 2$. For $k=0$ the assertion is obvious, and if it holds for some $k$, and $d_{k} \leq$ ord $p$, then by (3) and (6) we obtain $d_{k+1} \geq 2^{k+1}$ and $w_{k+1} \geq 2^{k}$, and if $d_{k}>$ ord $p$, then the preceding lemma implies $w_{k+1} \leq$ ord $p$ and (6) gives $w_{k+1} \geq 3 \cdot 2^{k-1}>2^{k}$.

In case $p=2$ the argument is the same, except that one uses (7) instead of $(6)$.

Using (4), (5) and Lemma 8 one immediately obtains the assertion of the Proposition.

By the Proposition, Lemma 3 and Lemma 6 we get Theorem 1.

7. Proof of Corollary 1 . Let $P$ be a prime ideal over $2 \mathbb{Z}_{K}$, let $f$ be its degree, $e$ its ramification index, and $R=\left(\mathbb{Z}_{K}\right)_{P}$ the corresponding localization. Clearly the cycle lengths in $\mathbb{Z}_{K}$ cannot exceed the maximal cycle length in $R$. So in particular $N(P)=2^{f}$, ord $2=e$ and $f \cdot e \leq N=$ $[K: Q]$. By using Theorem 1(i) one deduces $\alpha \leq e$; and as $e \leq N$ we conclude that the cycle lengths are bounded by

$$
2^{f}\left(2^{f}-1\right) 2^{e} \leq 2^{N / e}\left(2^{N / e}-1\right) 2^{e} \leq 2^{N+1}\left(2^{N}-1\right) .
$$

8. Pr o of of Corollary 2. As we have seen in the proof of Theorem 1 we can write $k=a_{1} b_{1} c_{1}=a_{2} b_{2} c_{2}$ where $a_{i} \leq N\left(P_{i}\right), b_{i} \mid\left(N\left(P_{i}\right)-1\right)$, and $c_{i}$ is a power of $p_{i}=\operatorname{char} R / P_{i}$. So

$$
c_{1}\left|a_{2} b_{2} c_{2} \Rightarrow c_{1}\right| a_{2} b_{2} \Rightarrow k \leq a_{1} b_{1} a_{2} b_{2} \text {. }
$$

\section{PROOF OF THEOREM 2}

9. We start with the non-existence assertion.

LEMMA 9. (i) If $y_{0}, \ldots, y_{p-1}$ is a $(*)$-cycle in $\mathbb{Z}_{p}$, and $v\left(y_{1}-y_{0}\right)=p^{-d}$ then $(p-2) d \leq 1$.

(ii) If $p>3$ then there are no $(*)$-cycles of length $p$ in $\mathbb{Z}_{p}$. In $\mathbb{Z}_{3}$ there are no $(*)$-cycles of length 9 and in $\mathbb{Z}_{2}$ there are no $(*)$-cycles of length 4. 
Proof. (i) Let $y_{0}, y_{1}, \ldots, y_{p-1}$ be a $(*)$-cycle for $F(X)=a_{p-1} X^{p-1}+$ $\ldots+a_{0}$ and $v\left(y_{1}-y_{0}\right)=p^{-d}, d \geq 1$. In view of Lemma 1(ii) one can assume $y_{i}=p^{d} z_{i}$ for $i=0,1, \ldots, p-1$, with $z_{0}=0, z_{1}=1$.

Consider the linear system

$$
(S)=\left\{\begin{array}{l}
a_{0}+a_{1} y_{0}+\ldots+a_{p-1} y_{0}^{p-1}=y_{1} \\
\ldots \ldots \ldots \ldots \ldots \ldots \ldots \ldots \ldots \ldots \\
a_{0}+a_{1} y_{p-1}+\ldots+a_{p-1} y_{p-1}^{p-1}=y_{0} .
\end{array}\right.
$$

If $\delta$ denotes its determinant, then $v(\delta)=p^{-d p(p-1) / 2}$ by Lemma $1(\mathrm{v})$ and we get

$$
\begin{aligned}
& p^{d p(p-1) / 2}\left|\begin{array}{ccccc}
1 & y_{0} & \ldots & y_{0}^{p-2} & y_{1} \\
\ldots & \ldots & \ldots & \ldots \ldots \ldots \\
1 & y_{p-1} & \ldots & y_{p-1}^{p-2} & y_{0}
\end{array}\right| \quad \text { and } \\
& p^{d(p-2)}|| \begin{array}{ccccc}
1 & z_{0} & \ldots & z_{0}^{p-2} & z_{1}-z_{0}-1 \\
\ldots \ldots \ldots \ldots \ldots \ldots \ldots \ldots \ldots \ldots \ldots \ldots & \ldots \ldots \ldots \\
1 & z_{p-1} & \ldots & z_{p-1}^{p-2} & z_{0}-z_{p-1}-1
\end{array} \mid=\Delta, \quad \text { say . }
\end{aligned}
$$

Since by Lemma $4, F^{\prime}(0) \equiv 1(\bmod p)$, Lemma 5 gives $z_{i} \equiv i(\bmod p)$ $(i=0,1, \ldots)$ and thus

with

$$
\Delta_{k}=\left|\begin{array}{cccc}
1 & z_{0} & \ldots & z_{0}^{p-2} \\
\ldots & \ldots \ldots & \ldots & \ldots \\
1 & z_{k-1} & \ldots & z_{k-1}^{p-2} \\
1 & z_{k+1} & \ldots & z_{k+1}^{p-2} \\
\ldots \ldots \ldots \ldots \ldots & \ldots \ldots \\
1 & z_{p-1} & \ldots & z_{p-1}^{p-2}
\end{array}\right| \equiv(-1)^{k} c(\bmod p)
$$

$$
c=\frac{1}{(p-1) !} \prod_{0 \leq i<j \leq p-1}(j-i) \not \equiv 0(\bmod p) .
$$

If we had $(p-2) d \geq 2$ then $p^{2} \mid \Delta$. But

$$
\Delta=\sum_{k=0}^{p-1}(-1)^{k}\left(z_{k+1}-z_{k}-1\right) \Delta_{k},
$$

and since $\Delta_{k}=(-1)^{k} c+p \alpha_{k}$ with a suitable $\alpha_{k} \in \mathbb{Z}_{p}$ we get

$$
\begin{aligned}
\Delta & =c \sum_{k=0}^{p-1}\left(z_{k+1}-z_{k}-1\right)+p \sum_{k=0}^{p-1}(-1)^{k}\left(z_{k+1}-z_{k}-1\right) \alpha_{k} \\
& \equiv-p c \not \equiv 0\left(\bmod p^{2}\right),
\end{aligned}
$$

since $z_{k+1}-z_{k}-1 \equiv 0(\bmod p)$ for $k=0,1, \ldots, p-1$, and this is a contradiction. 
(ii) In case $p=2,3$ the assertion results from Theorem 1 and for $p>3$ it is an immediate consequence of (i).

Lemma 10. There are no $(*)$-cycles of length 6 in $\mathbb{Z}_{3}$.

Proof. The preceding lemma shows that if $0, z_{1}, z_{2}$ is a $(*)$-cycle in $\mathbb{Z}_{3}$, then $v\left(z_{1}\right)=1 / 3$. Let $0, y_{1}, \ldots, y_{5}$ be a $(*)$-cycle of length 6 in $\mathbb{Z}_{3}$ realized by the polynomial $F(X)=a_{5} X^{5}+\ldots+a_{0}$. Lemma 9(i) implies $v\left(y_{2}\right)=v\left(y_{4}\right)=1 / 3$. This implies $v\left(y_{1}\right)=1 / 3$ and $v\left(y_{3}\right)<1 / 3$ since there are only three residue classes mod 3 . Now Lemma 1 shows that it suffices to consider the cycle

$$
0,3,6+9 c, 9 \cdot 3^{D} u, 3+9 \cdot 3^{D} v, 6+9 c+3^{D} w,
$$

with $D \geq 0$ and $3 \nmid u v w$.

Considering again the system $(S)$ with determinant $\delta$ we get $v(\delta)=$ $3^{-18-3 D}$. Put $\mathbf{A}=2+3 c+3^{1+D} w, \mathbf{B}=2+3 c$. Observe that $a_{2} \in \mathbb{Z}_{3}$ implies the divisibility of the determinant

$$
\left|\begin{array}{cccccc}
1 & 0 & 1 & 0 & 0 & 0 \\
1 & 1 & 2+3 c & 1 & 1 & 1 \\
1 & 2+3 c & 3^{1+D} u & (2+3 c)^{3} & (2+3 c)^{4} & (2+3 c)^{5} \\
0 & 3^{1+D} u & 3^{1+D} v & \left(3^{1+D} u\right)^{3} & \left(3^{1+D} u\right)^{4} & \left(3^{1+D} u\right)^{5} \\
0 & 3^{1+D} v & 3^{1+D} w & \left(1+3^{1+D} v\right)^{3}-1 & \left(1+3^{1+D} v\right)^{4}-1 & \left(1+3^{1+D} v\right)^{5}-1 \\
0 & 3^{1+D} w & -3^{1+D} u & \mathbf{A}^{3}-\mathbf{B}^{3} & \mathbf{A}^{4}-\mathbf{B}^{4} & \mathbf{A}^{5}-\mathbf{B}^{5}
\end{array}\right|
$$

by $3^{4+3 D}$. All elements of the last three lines of this determinant are divisible by $3^{1+D}$, hence

$$
3|| \begin{array}{cccccc}
1 & 0 & 1 & 0 & 0 & 0 \\
1 & 1 & 2 & 1 & 1 & 1 \\
1 & 2 & 0 & 2 & 1 & 2 \\
0 & u & v & 0 & 0 & 0 \\
0 & v & w & 0 & v & 2 v \\
0 & w & -u & 0 & 2 w & 2 w
\end{array}|, \quad 3| v u+w^{2} \quad \text { and } \quad 3 \mid u v+1
$$

Now $a_{3} \in \mathbb{Z}_{3}$ implies

$$
3^{5+3 D}|| \begin{array}{cccccc}
1 & 0 & 0 & 1 & 0 & 0 \\
1 & 1 & 1 & 2+3 c & 1 & 1 \\
1 & 2+3 c & (2+3 c)^{2} & 3^{1+D} u & (2+3 c)^{4} & (2+3 c)^{5} \\
0 & 3^{1+D} u & \left(3^{1+D} u\right)^{2} & 3^{1+D} v & \left(3^{1+D} u\right)^{4} & \left(3^{1+D} u\right)^{5} \\
0 & 3^{1+D} v & \left(1+3^{1+D} v\right)^{2}-1 & 3^{1+D} w & \left(1+3^{1+D} v\right)^{4}-1 & \left(1+3^{1+D} v\right)^{5}-1 \\
0 & 3^{1+D} w & \mathbf{A}^{2}-\mathbf{B}^{2} & -3^{1+D} u & \mathbf{A}^{4}-\mathbf{B}^{4} & \mathbf{A}^{5}-\mathbf{B}^{5}
\end{array} \mid
$$

and here again all elements of the last three rows are divisible by $3^{1+D}$, 
hence

$$
\begin{gathered}
3\left|\begin{array}{cccccc}
1 & 0 & 0 & 1 & 0 & 0 \\
1 & 1 & 1 & 2 & 1 & 1 \\
1 & 2 & 1 & 0 & 1 & 2 \\
0 & u & 0 & v & 0 & 0 \\
0 & v & 2 v & w & v & 2 v \\
0 & w & w & -u & 2 w & 2 w
\end{array}\right|, \\
3 \mid u\left(w^{2}-v(u+w)\right)-v \cdot v \cdot w
\end{gathered}
$$

and

$$
3 \mid u-v-w(1+u v)
$$

but since $3 \mid u v+1$ we get $u \equiv v(\bmod 3)$, and $3 \mid u^{2}+1$, a contradiction.

10. Now we construct cycles with lengths listed in Theorem 2 and start with $(*)$-cycles. Obviously for any $p$ the polynomial $-X+p$ realizes the (*)-cycle $0, p$ of length 2 in $\mathbb{Z}_{p}$, and the polynomial $-\frac{1}{2} X(X-3)+X+3$ realizes the $(*)$-cycle $0,3,6$ of length 3 in $\mathbb{Z}_{3}$, and this settles the exceptional cases in Theorem 2(i). The remaining cases of (i) are covered by the following lemma, which gives slightly more than needed:

LEMMA 11. If $R$ is a complete discrete valuation domain of zero characteristic with prime ideal $P=\pi R$ and finite residue field of $N(P)$ elements, then there exists a $(*)$-cycle of any length dividing $N(P)-1$.

Proof. In view of Lemma 1(iii) it suffices to find a cycle of length $N(P)-1$. Clearly we may assume $N(P)>2$. Denote by $g_{0}$ any primitive root $\bmod P$ and put

$$
W(X)=1+X+X^{2}+\ldots+X^{N(P)-2} .
$$

Clearly $W\left(g_{0}\right) \equiv 0(\bmod P)$, and Hensel's lemma shows the existence of a root $g \in R$ of $W$. The polynomial $g X+\pi$ realizes the cycle

$$
0, \pi,(1+g) \pi, \ldots,\left(1+g+g^{2}+\ldots+g^{N(P)-3}\right) \pi
$$

of length $N(P)-1$.

The proof of part (ii) of Theorem 2 in the exceptional cases $p=2,3$ follows from the following examples of cycles:

(a) $F(X)=-\frac{2}{3} X(X-1)(X-2)+X+1$ has the cycle $0,1,2,3$ of 4 elements in $\mathbb{Z}_{2}$, in $\mathbb{Z}_{3}$,

(b) $F(X)=-\frac{1}{4} X^{3}+\frac{1}{2} X^{2}+\frac{7}{4} X+1$ has the cycle $0,1,3,4$ of 4 elements

(c) $F(X)=-\frac{1}{20} X(X-1)(X-2)(X-3)(X-4)+X+1$ has the cycle $0,1,2,3,4,5$ of 6 elements in $\mathbb{Z}_{3}$, 
(d) $F(X)=-\frac{9}{8 !} X(X-1)(X-2)(X-3)(X-4)(X-5)(X-6)(X-7)$ $+X+1$ has the cycle $0,1,2,3,4,5,6,7,8$ of 9 elements in $\mathbb{Z}_{3}$.

In the remaining cases the assertion (ii) is a consequence of the following lemma:

LEMMA 12. If $R$ is a complete discrete valuation domain of zero characteristic with prime ideal $P=\pi R$ and finite residue field of $N(P)$ elements, and there exists in $R$ a (*)-cycle of length $m$, then for each $r=$ $0,1, \ldots, N(P)-1$ there exists in $R$ a cycle of length $(1+r) m$.

Proof. Let $M=(1+r) m$ and let $a_{0}=0, a_{1}, \ldots, a_{r}$ be elements of $R$ lying in different cosets $(\bmod P)$. Moreover, let $y_{0}=0, y_{1}, \ldots, y_{m-1}$ be a (*)-cycle realized by a polynomial $F$. For $n=1,2, \ldots$ put

$$
\begin{aligned}
W_{n}(X)= & \left(1-\left(X-a_{r}\right)^{N(P)^{n}(N(P)-1)}\right) F\left(X-a_{r}\right) \\
& +\sum_{j=0}^{r-1}\left(\left(1-\left(X-a_{j}\right)^{N(P)^{n}(N(P)-1)}\right)\left(X+a_{j+1}-a_{j}\right)\right) .
\end{aligned}
$$

Thus $W_{n}^{l(1+r)+j}\left(y_{0}\right) \equiv y_{l}+a_{j}\left(\bmod P^{n+1}\right)$ for $j=0,1, \ldots, r$.

Let

$$
L_{n}(X)=\sum_{i=0}^{M-1} a_{i}^{(n)} X^{i}
$$

be the remainder of the division of $W_{n}(X)$ by the polynomial

$$
X \prod_{j=1}^{M-1}\left(X-W_{n}^{j}(0)\right) .
$$

A simple recurrence argument gives $L_{n}^{j}(0)=W_{n}^{j}(0)(j=1,2, \ldots, M)$. Choose now a subsequence $n_{1}, n_{2}, \ldots$ so that the limits

$$
c_{i}=\lim _{k \rightarrow \infty} a_{i}^{\left(n_{k}\right)}
$$

exist for each $i=0,1, \ldots, M$, and put

$$
L(X)=\sum_{i=0}^{M-1} c_{i} X^{i} .
$$

Then

$$
L^{l(1+r)+j}\left(y_{0}\right)=\lim _{k \rightarrow \infty} L_{n_{k}}^{l(1+r)+j}\left(y_{0}\right)=\lim _{k \rightarrow \infty} W_{n_{k}}^{l(1+r)+j}\left(y_{0}\right)=y_{l}+a_{j}
$$

and thus the polynomial $L$ realizes a cycle of $M$ elements.

Note that the assertions of Lemmas 11 and 12 remain true also if $R$ is not complete. Indeed, let $S$ be the completion of $R$ and $x_{0}, x_{1}, \ldots, x_{m-1}$ a cycle in $S$. Choose a sequence $y_{0}, y_{1}, \ldots, y_{m-1}$ with $v\left(y_{i}-x_{i}\right)$ sufficiently small 
for all $i$. It follows from the Lagrange interpolation formula that the unique polynomial $F$ of degree not exceeding $m-1$ which satisfies $F\left(y_{i}\right)=y_{i+1}$ for $i=0,1, \ldots, m-2$ and $F\left(y_{m-1}\right)=y_{0}$ has its coefficients in $R$.

11. Proof of Corollary 3. It suffices to observe that every prime congruent to $1(\bmod M)$ splits in the $M$ th cyclotomic field and apply Theorem 2 .

\section{Reference}

[1] W. Narkiewicz, Polynomial cycles in algebraic number fields, Colloq. Math. 58 (1989), 151-155.

MATHEMATICAL INSTITUTE

UNIVERSITY OF WROCŁAW

PL. GRUNWALDZKI $2 / 4$

50-384 WROCŁAW, POLAND 